\title{
Verteilungsmuster psychopathologischer Störungen und deren Bezug zum psychosozialen Hintergrund bei Erstvorstellungen an der Terminambulanz für Kinder- und Jugendpsychiatrie an der Universitätsklinik Salzburg
}

\author{
Christine Skrivanek (D) - Elisabeth Marte · Kornelius Winds · Leonhard Thun-Hohenstein · Belinda Plattner
}

Eingegangen: 5. März 2019 / Angenommen: 27. Mai 2019 / Online publiziert: 13. Juni 2019

(C) Der/die Autor(en) 2019

\begin{abstract}
Zusammenfassung
Grundlagen Die vorliegende Studie beschreibt die Prävalenz psychischer Störungen und psychosozialer Belastungen einer Kohorte von geplant erstvorstelligen PatientInnen an einer kinder- und jugendpsychiatrischen Spitalsambulanz.

Methodik Einhundertdreiundachzig PatientInnen im Alter von 10-19 Jahren wurden mittels Mini International Neuropsychiatric Interview for Children and Adolescents und Multidimensional Clinical Screening Inventory untersucht. Pearson's Chi-Square Tests wurden angewandt, um alters- und geschlechtsspezifische Unterschiede in der Häufigkeit psychiatrischer Störungsbilder sowie deren Zusammenhang mit den psychosozialen Hintergründen zu untersuchen.

Ergebnisse Die meisten PatientInnen (86,9\%) präsentierten sich mit einer psychischen Störung, wobei $72,4 \%$ bereits außerhalb des Krankenhauses psychische Behandlung in Anspruch genommen haben. Es zeigten sich keine geschlechtsspezifischen Unterschiede bei Erkrankungshäufigkeit und Lebensalter. Internalisierende Störungen traten häufiger bei äl-
\end{abstract}

C. Skrivanek, M. Sc. $(\bowtie) \cdot$ Dr. med. univ. E. Marte ·

Dr. med. univ. K. Winds · Prof. Dr. L. Thun-Hohenstein

PD Dr. B. Plattner

Universitätsklinik für Kinder- und Jugendpsychiatrie, Christian Doppler Klinik, Paracelsus Medizinische

Privatuniversität, Ignaz-Harrer-Straße 79, 5020 Salzburg, Österreich

christine.skrivanek@gmail.com

PD Dr. B. Plattner

belinda.plattner@pmu.ac.at

PD Dr. B. Plattner

Ordination für Kinder- und Jugendpsychiatrie, Hans

Kappacherstraße 15, 5600 St. Johann im Pongau, Österreich

Paracelsus Medizinische Privatuniversität,

Strubergasse 21, 5020 Salzburg, Österreich teren und externalisierende Störungen häufiger bei männlichen sowie jüngeren PatientInnen auf. 65,2\% zeigten Symptome mindestens einer komorbiden Störung. Jungen und jene mit mindestens einer externalisierenden Störung wiesen mehr Probleme in der Schule auf. Jungen waren öfter von extra-familiärer Gewalt, Mädchen öfter von sexuellem Missbrauch betroffen. Mädchen und PatientInnen mit internalisierenden Störungen zeigten häufiger selbstverletzendes Verhalten und Selbstmordversuche. Die Kombination von externalisierenden und internalisierenden Störungen begünstigte Drogenkonsum. Jungen verbrachten ihre Freizeit häufiger am PC.

Schlussfolgerungen Die Ergebnisse zeigen, dass einige Psychopathologien in Zusammenhang mit bestimmten psychosozialen Belastungen stehen. Vor allem Jungen zeigen sich im Schulsystem vulnerabel. Die im Zusammenhang mit psychischer Störung beobachteten psychosozialen Belastungen und die vorhandene Bereitschaft der PatientInnen, extramurale psychische Behandlungen in Anspruch zu nehmen, unterstützen die Notwendigkeit eines vernetzen Arbeitens an der Kinder- und Jugendpsychiatrie mit Einbezug der Eltern, Schulen und der Kinder- und Jugendhilfe.

Schlüsselwörter Kinder- und Jugendpsychiatrie · Psychopathologie - Psychosozialer Hintergrund . Internalisierende Störungen - Externalisierende Störungen 
Patterns of psychopathology and psychosocial background of patients at first presentation at the child and adolescent psychiatric outpatient department of the University Clinic Salzburg

\section{Summary}

Background This study examines the prevalence of psychiatric disorders and psychosocial background of outpatients at an Austrian child and adolescent psychiatry department.

Methods One hundred eighty-three patients were examined using the Mini International Neuropsychiatric Interview for Children and Adolescents and the Multidimensional Clinical Screening Inventory. Pearson's chi-square tests were used to explore the prevalence of psychiatric disorders between gender and agegroups and their relationship with the psychosocial background of patients.

Results Most patients (86.9\%) had at least one psychiatric disorder. Almost three quarters (72.4\%) of these patients made use of extramural facilities. Boys and girls did not differ in age or presence of a disorder. Internalising disorders were more common among older patients. Externalising disorders were more common among boys and younger patients. $65.2 \%$ of patients suffered from at least one co-morbid disorder. Boys and those with at least one externalising disorder showed more problems at school. Girls were victims of sexual assault more often, but boys experienced more extra-familial physical abuse. Girls and those with internalising disorders showed more selfharming and suicidal behaviour. A combination of internalising and externalising disorders correlated with increased drug consumption. Boys spent more time using a computer.

Conclusions The results show that particular psychopathologies often appear in connection with certain psychosocial burdens. Especially boys show increased vulnerability in relation to school problems. The willingness of patients to make use of extramural points of contact should guide future policies to consider therapeutic options encompassing parents, schools and welfare institutions for children prone to the development of psychiatric symptoms due to psychosocial risk.

Keywords Child and adolescent psychiatry . Psychopathology · Psychosocial background . Internalising disorders · Externalising disorders

\section{Abkürzungen}

ACEs

ADHS

Adverse Childhood Experiences

DSM-IV Aufmerksamkeitsdefizit-/Hyperaktivitätsstörung

Diagnostic and Statistical Manual of Mental Health, Fourth Edition

ICD-10 International Statistical Classification of Diseases and Related Health Problems 10th Revision
MCSI Multidimensional Clinical Screening Inventory

MHAT-Study Mental Health in Austrian Teenagers Study

MINI-KID Mini International Neuropsychiatric Interview for Children and Adolescents

PTBS Posttraumatische Belastungsstörung

\section{Hintergrund}

Studien zu psychischen Krankheitsbildern und psychosozialen Hintergründen bei Kindern und Jugendlichen in Österreich sind rar. Sie sind aber wichtig, um eine evidenzbasierte Arbeit und Schwerpunktsetzungen im kinder- und jugendpsychiatrischen Tätigkeitsfeld zu ermöglichen.

Eine groß angelegte, rezente Studie (Mental Health in Austrian Teenagers-Study; MHAT, [1]) untersuchte eine Kohorte von 3615 Kindern und Jugendlichen in österreichischen Schulen als auch 292 TeilnehmerInnen aus einer stationären klinischen Stichprobe. Die Studie ergab eine Punkt- und Lebenszeitprävalenzrate für psychische Erkrankungen von 23,9\% bzw. $35,8 \%$. Vierzig Prozent aller Kinder und Jugendlichen der Gesamtstichprobe, welche einer Störungskategorie zugeordnet werden konnten, hatten mindestens eine komorbide Störung. Das Geschlecht der Kinder und Jugendlichen war nicht prädiktiv für die Prävalenz einer oder mehrerer psychischer Erkrankungen. Junge Männer litten allerdings häufiger an einer Aufmerksamkeitsdefizit-/Hyperaktivitätsstörung (ADHS) $(15,4 \%$ vs. $5,2 \%)$ sowie an Impulskontrollund Verhaltensstörungen (7,44\% vs. 1,26\%). Mädchen hingegen litten häufiger an Angststörungen (19,53\% vs. 9,52\%), traumaassoziierten Störungen $(4,94 \%$ vs. $1,30 \%)$, Essstörungen (5,47\% vs. $0,64 \%)$, suizidalen Verhaltensstörungen $(2,53 \%$ vs. $0,33 \%)$ und zeigten als Einzige selbstverletzendes Verhalten $(3,49 \%$ vs. $0 \%)$ [1].

Die klinische Stichprobe der MHAT-Study zeigte, verglichen mit ProbandInnen ohne eine messbare Störung, dass sie häufiger weiblich waren, bei alleinerziehenden Eltern lebten, mehr chronische und psychiatrische Erkrankungen in der engeren Familie hatten und öfter traumatischen Erlebnissen sowie Missbrauch ausgesetzt waren [1]. Kinder und Jugendliche mit einer psychischen Erkrankung haben in anderen Studien weiters häufiger sonderpädagogischen Förderbedarf gezeigt und blieben öfter unerlaubt der Schule fern [2].

Es besteht ein deutlicher Zusammenhang zwischen adversiven Kindheitserfahrungen (Adverse Childhood Experiences - ACEs) und psychischer Erkrankung. Mit steigender Anzahl an ACEs, verdreifacht sich im Durchschnitt die Wahrscheinlichkeit für das Auftreten komorbider Störungen [3]. In einer U.S.-Amerikanischen Stichprobe von 2293 15- bis 17-Jährigen gaben 26,6\% der 17-jährigen Mädchen an, Opfer von sexueller Gewalt gewesen zu sein - im Vergleich zu 5,1\% 
der männlichen Gleichaltrigen [4]. Sexuelle Gewalt sowie physische Bestrafungen erhöhen im Gegenzug die Wahrscheinlichkeit für einen Suizidversuch [5]. Vollendeter Suizid ist ein überwiegend männliches Phänomen [6]. Hingegen tätigen mehr Mädchen Suizidversuche. Dies könnte Ausdruck einer höheren Prävalenz internalisierender Erkrankungen sein [7].

In Bezug auf psychosoziale Hintergründe besteht ein kumulativer Effekt von negativen Erlebnissen in Bezug auf Psychopathologien. Bielas et al. [8] fanden, dass die Anzahl potentiell traumatisierender Erlebnisse sowohl mit dem Auftreten von Irritabilität zusammenhängt als auch prädiktiv für die Prävalenz depressiver Erkrankungen, Suizidalität, posttraumatischer Belastungsstörung (PTBS) und Angststörungen ist. Diese internalisierenden Störungen zeigten in der Vergangenheit häufig einen Zusammenhang mit akkumulierter psychosozialer Belastung [9, 10]. Irritabilität wiederum, als Ausdruck der emotionalen Dysregulation, korrelierte mit Depression, Suizidalität, Störungen des Sozialverhaltens, Substanzmissbrauch und ADHS. Zusätzlich ist die Erstmanifestation einer Depression während der Adoleszenz sowohl auf Grund der akkumulierten ACEs, als auch neurobiologischer Umbauprozesse besonders häufig. [11].

Diese Forschungsergebnisse legen nahe, dass gewisse psychische Erkrankungen aus ACEs und den daraus folgenden emotionalen Störungen resultieren. Die AutorInnen argumentieren daher für eine spezifische emotionsregulative Behandlung der betroffenen PatientInnen [8].

In Bezug auf schulische Auffälligkeiten haben ältere Studien gezeigt, dass sich Jungen mit ADHS häufiger in Behandlung befinden als Mädchen, begünstigt durch die hauptsächlich externalisierende Ausprägung der Erkrankung bei männlichen Patienten und der daraus resultierenden schlechteren Beurteilung durch Lehrpersonal [12]. Die häufigere Externalisierung psychischer Erkrankungen bei Jungen deutet auf eine gesteigerte Vulnerabilität in Bezug auf schulische Leistungen hin.

Die vorliegende Studie beschreibt die bisher wenig untersuchte Prävalenz psychischer Erkrankungen und den psychosozialen Hintergrund von ambulant vorstelligen Kindern und Jugendlichen. Bisherige Studien aus Österreich beziehen sich mehrheitlich auf die Versorgungssituation der Kinder- und Jugendpsychiatrien im Allgemeinen [13, 14]. Es ist insbesondere von Interesse, welche Bedürfnisse die Kinder und Jugendlichen, die ambulant versorgt werden, aus psychopathologischer und psychosozialer Sicht aufweisen.

\section{Methoden}

Alle Kinder und Jugendlichen, die von 12.11.2013 bis 27.11.2015 an der Terminambulanz der Universitätsklinik für Kinder- und Jugendpsychiatrie der Christian Doppler Klinik Salzburg erstmals vorstellig wurden, wurden mit standardisierten Untersuchungsin- strumenten diagnostiziert. Von den 183 Kindern und Jugendlichen erfüllten 154 im Alter von 10-19 Jahren die Einschlusskriterien für die vorliegende Studie.

Einschlusskriterien waren eine ausreichende Kenntnis der deutschen Sprache in Wort und Schrift, um Selbstbeurteilungsfragebögen selbstständig ausfüllen und diagnostische Interviews verstehen und beantworten $\mathrm{zu}$ können. Ausschlusskriterien waren Umstände, welche das korrekte Verstehen und Ausfüllen der Fragebögen beziehungsweise Interviews unmöglich machten, wie zum Beispiel akute psychiatrische Erkrankungen mit psychotischen Symptomen oder eine intellektuelle Minderbegabung. Die Erstuntersuchung wurde von Fach- und Assistenzärztinnen für Kinder- und Jugendpsychiatrie und Psychotherapie durchgeführt.

Bei Vorstellung an der Ambulanz für Kinder- und Jugendpsychiatrie wurden die demographischen Daten Geschlecht und Alter erhoben.

\section{Mini International Neuropsychiatric Interview for Children and Adolescents (MINI-KID)}

Das MINI-KID stellt ein strukturiertes klinisch diagnostisches Interview dar, welches auf eine für Kinder und Jugendliche verständliche Art und Weise psychiatrische Störungen nach DSM-IV (Diagnostic and Statistical Manual of Mental Health, Fourth Edition; [15]) und ICD 10 (International Classification of Diseases, 10. Revision; [32]) untersucht. Angelehnt an die Erwachsenenform des Interviews, welche unter anderem affektive und psychotische Störungen, sowie Angst-, Substanz- und Abhängigkeitsstörungen untersucht, beurteilt das MINI-KID zusätzlich relevante psychiatrische Störungen bei Kindern und Jugendlichen, wie beispielsweise Trennungsangst, Aufmerksamkeitsdefizit-/Hyperaktivitätsstörung (ADHS) und Störungen des Sozialverhaltens [16].

\section{Multidimensional Clinical Screening Inventory (MCSI)}

In dieser Studie wurde eine für nicht delinquente PatientInnen adaptierte Version des Multidimensional Clinical Screening Inventory for Delinquent Juveniles verwendet, um die psychosozialen Hintergründe der PatientInnen zu untersuchen [17]. In Anlehnung an die geläufige klinische kinder- und jugendpsychiatrische Anamnese werden mittels dieses halbstrukturierten Interviews schulische Auffälligkeiten, frühere psychische Behandlungen ja/nein, (ambulant, stationär, kinder- und jugendpsychiatrisch, psychologisch, psychotherapeutisch), psychiatrische Familienanamnese und das Freizeitverhalten erhoben. Des Weiteren werden intra- und extrafamiliäre Traumata (sexueller, körperlicher und emotionale Missbrauch, körperliche und emotionale Vernachlässigung, Trennung und Verlust) sowie schädlicher Gebrauch von Alkohol und Drogen erhoben [17]. 


\section{Statistische Auswertung}

Die Daten wurden mittels Statistical Package for Social Sciences Version 22 (SPSS Inc, Chicago, IL) analysiert. Mit Hilfe eines t-Tests wurde ermittelt, ob es einen Unterschied im Alter bei Erstvorstellung zwischen Mädchen und Jungen gab. Die Homoskedastizität der Daten wurde bestätigt durch einen Levene-Test. Pearson's Chi-Square Tests wurden angewandt, um dichotome Daten auf mögliche Unterschiede in der Häufigkeit psychiatrischer Störungsbilder sowie deren $\mathrm{Zu}$ sammenhang psychosozialen Hintergründen $\mathrm{zu}$ untersuchen. Bei einer erwarteten Häufigkeit von $n<5$ wurde der Exakte Test nach Fisher verwendet, welcher eine geeignete Methode darstellt, um $2 \times 2$ Kontingenztabellen auch bei kleinen Stichproben zu erstellen [18, 19].

Die psychiatrischen Störungskategorien des MINIKID wurden als Erstes in Anlehnung an das ICD-10 zu Verhaltensstörungen (Störung des Sozialverhaltens, oppositionelles Trotzverhalten), Aufmerksamkeits-/ Hyperaktivitätsstörungen (Aufmerksamkeitssubtyp, Hyperaktivitätssubtyp, kombiniert), posttraumatische Belastungsstörungen, Angst-/Panikstörungen (Panikstörung, Agoraphobie, Trennungsangst, spezifische Phobie, generalisierte Angststörung), Essstörungen (Anorexia nervosa, Bulimia nervosa), Suizidalität, Depression/Dysthymie und Anpassungsstörungen (depressive Anpassungsstörung, ängstliche Anpassungsstörung, Anpassungsstörung mit Verhaltensstörung, soziale Anpassungsstörung) zusammengefasst [15]. Das Patientenkollektiv wurde folglich in Bezug auf diese Störungskategorien getrennt nach Geschlecht untersucht. Auf Grund der Trennung zwischen Kindern und Jugendlichen, wurden alle Daten von PatientInnen, unter und ab 14 Jahre separat analysiert.

Die ursprünglichen Störungskategorien des MINIKID wurden danach außerdem in internalisierende und externalisierende Störungen unterteilt. Die internalisierenden Störungen setzten sich aus posttraumatischer Belastungsstörung, Angststörungen, $\mathrm{Pa}$ nikstörung, Zwangsstörung, Essstörung, Suizidalität, Depression, Dysthymie und Anpassungsstörungen zusammen. Die externalisierenden Störungen wurden gruppiert in Alkohol- und Substanzmissbrauch und Abhängigkeit, ADHS, Störungen des Sozialverhaltens und oppositionellem Trotzverhalten. Diese wurden in weiterer Folge auf einen möglichen Zusammenhang mit Verhaltensauffälligkeiten in der Schule, psychiatrischer Geschichte, intra- und extrafamiliären sowie freizeitanamnestischen Variablen des MCSI untersucht.

\section{Ergebnisse}

Die allgemeine Auswertung der Psychopathologieprävalenz aller PatientInnen ergab, dass 119 (86,9\%) die Kriterien für mindestens eine Störungskategorie erfüllten. Die Anzahl an PatientInnen wird in Tab. 1 getrennt nach Geschlecht und Alter zusammengefasst. Mädchen und Burschen, sowie PatientInnen unter und ab 14 Jahren, unterschieden sich nicht signifikant im Auftreten psychischer Erkrankungen (siehe Tab. 1). Das Durchschnittsalter aller PatientInnen betrug 14,7 [SD = 1,9] Jahre. Kein Unterschied fand sich in Bezug auf das Alter von männlichen und weiblichen PatientInnen bei Erstvorstellung in der Ambulanz. Mädchen waren im Durchschnitt $14,84[\mathrm{SD}=1,76]$, und Jungen 14,56 [SD=2] Jahre alt; $\mathrm{t}(152)=-0,92 ; p=0,359$.

Tab. 2 zeigt die Unterschiede in der Häufigkeit der Störungskategorien zwischen weiblichen und männlichen PatientInnen. So litten signifikant mehr Jungen an Verhaltensstörungen und Aufmerksamkeits- und/oder Hyperaktivitätsstörungen. Mädchen hingegen litten öfter unter posttraumatischen Belastungsstörungen, Angst und/oder Panikstörungen, Essstörungen, Suizidalität, und Depression und/oder Dysthymie. Im Weiteren ergaben sich Unterschiede in der Häufigkeit der verschiedenen Erkrankungen zwischen PatientInnen unter und ab 14 Jahren. PatientInnen jünger als 14 Jahre präsentierten sich öfter mit Verhaltensstörungen und Aufmerksamkeits- und/ oder Hyperaktivitätsstörungen. Ältere PatientInnen litten hingegen öfter unter Suizidalität und Depression und/oder Dysthymie (siehe Tab. 2).

In der gesamten Stichprobe hatten $65,2 \%$ aller PatientInnen mindesten eine komorbide Störung. Hinsichtlich des Geschlechts der ProbandInnen fand sich eine erhöhte Anzahl einzelner Störungen bei Mädchen. In Bezug auf das Alter der PatientInnen zeigte sich, dass ProbandInnen unter 14 Jahren deutlich häufiger an einer Kombination aus zwei Störungen litten (siehe Tab. 2).

Nach Zusammenfassung aller externalisierender und internalisierender Störungskategorien gab es $19(13,7 \%)$ PatientInnen, welche keiner Störungskategorie zugeordnet werden konnten, 50 (36,2\%), welche ausschließlich internalisierende Störungen aufwiesen, 19 (13,7\%) zeigten nur externalisierende Störungen und 50 (36,2\%) wurden Störungen aus externalisierenden und internalisierenden Kategorien zugeordnet. Männliche Patienten zeigten im Vergleich $\mathrm{zu}$ weiblichen häufiger externalisierende Störungen $(69,6 \%$ vs. $37,0 \%), \chi 2(1)=14,081 ; p<0,001$.

Tab. 1 Anzahl an erstvorstelligen Patienten und Patientinnen, unter und ab 14 Jahre, sowie Anzahl an Erkrankten

\begin{tabular}{|c|c|c|c|c|c|c|}
\hline & Jungen & Mädchen & $p$ & Unter 14 Jahre & 14 Jahre oder älter & $p$ \\
\hline Anzahl insgesamt & $66(42,8 \%)$ & $88(57,1 \%)$ & - & $36(23,3 \%)$ & $118(76,6 \%)$ & - \\
\hline Mind. 1 Störungskategorie & $50(89,3 \%)$ & $69(85,2 \%)$ & 0,485 & $30(93,8 \%)$ & $89(84 \%)$ & 0,159 \\
\hline
\end{tabular}


Tab. 2 Vergleich der Häufigkeit psychiatrischer Störungen und der Anzahl an psychischen Störungskategorien pro Patientln bei Jungen und Mädchen und PatientInnen unter und ab 14 Jahren

\begin{tabular}{|c|c|c|c|c|c|c|c|}
\hline Störungen & $\begin{array}{l}\text { Gesamt } \\
N=138\end{array}$ & $\begin{array}{l}\text { Jungen } \\
N=57\end{array}$ & $\begin{array}{l}\text { Mädchen } \\
N=81\end{array}$ & $P$ & $\begin{array}{l}\text { Patientlnnen } \\
<14 \text { Jahre } \\
N=32\end{array}$ & $\begin{array}{l}\text { PatientInnen } \\
\geq 14 \text { Jahre } \\
N=106\end{array}$ & $p$ \\
\hline Verhaltensstörung & $38(27,5 \%)$ & $23(40,4 \%)$ & $15(18,5 \%)$ & $0,005^{\star \star}$ & $14(43,8 \%)$ & $24(22,6 \%)$ & $0,019^{\star}$ \\
\hline $\begin{array}{l}\text { Aufmerksamkeits-I } \\
\text { Hyperaktivitätsstörung }\end{array}$ & $47(34,1 \%)$ & $31(54,4 \%)$ & $16(29,8 \%)$ & $0,000^{\star *}$ & $16(50 \%)$ & $31(29,2 \%)$ & $0,03^{*}$ \\
\hline $\begin{array}{l}\text { Posttraumatische Belas- } \\
\text { tungsstörung }\end{array}$ & $35(25,4 \%)$ & $9(15,8 \%)$ & $26(32,1 \%)$ & $0,03^{*}$ & $9(28,1 \%)$ & $26(24,5 \%)$ & 0,682 \\
\hline Angst/Panikstörung & $43(31,2 \%)$ & $12(21,1 \%)$ & $31(38,3 \%)$ & $0,032^{*}$ & $6(18,8 \%)$ & $37(34,9 \%)$ & 0,084 \\
\hline Essstörung & $11(8,0 \%)$ & $0(0 \%)$ & $11(13,6 \%)$ & $0,003^{a, \star \star}$ & $3(9,4 \%)$ & $8(7,5 \%)$ & $0,717^{a}$ \\
\hline Suizidalität & $37(26,8 \%)$ & $8(14 \%)$ & $29(35,8 \%)$ & $0,04^{*}$ & $3(9,4 \%)$ & $34(32,1 \%)$ & $0,007^{a, *}$ \\
\hline Depression/Dysthymie & $44(31,9 \%)$ & $10(17,5 \%)$ & $34(42,0 \%)$ & $0,002^{\star *}$ & $4(12,5 \%)$ & $40(37,7 \%)$ & $0,009^{a, * *}$ \\
\hline Anpassungsstörung & $22(15,9 \%)$ & $12(21,2 \%)$ & $10(12,3 \%)$ & 0,169 & $8(25 \%)$ & $14(13,2 \%)$ & 0,110 \\
\hline Keine Störung & $19(13,8 \%)$ & $8(14 \%)$ & $11(13,6 \%)$ & 0,939 & $1(31, \%)$ & $18(17,0 \%)$ & $0,075^{a}$ \\
\hline Eine Störung & $29(21 \%)$ & $17(29,8 \%)$ & $12(14,8 \%)$ & $0,033^{*}$ & $9(28,1 \%)$ & $20(18,9 \%)$ & 0,260 \\
\hline Mehr als eine Störung & $89(65,9 \%)$ & $32(58,2 \%)$ & $57(71,3 \%)$ & 0,115 & $22(71 \%)$ & $67(63,8 \%)$ & 0,461 \\
\hline \multicolumn{8}{|c|}{$\begin{array}{l}{ }^{*} \mathrm{Ch}^{2} \text {-Test ist signifikant } p<0,05 \\
{ }^{*} \mathrm{Ch}^{2} \text {-Test ist signifikant } p<0,01 \\
{ }^{\mathrm{a} E} \text { Exakter Test nach Fisher bei erwartetem } n<5\end{array}$} \\
\hline
\end{tabular}

Tab. 3 Vergleich schulischer Auffälligkeiten zwischen Jungen und Mädchen suspendierung und am häufigsten Leistungsprobleme angaben. Mädchen gaben ebenso am seltensten Schulsuspendierungen, am häufigsten hingegen Probleme mit SchulkollegInnen an. Es zeigte sich, dass innerhalb der Kategorien Verhaltensauffälligkeiten, Raufhandel, Probleme mit Autoritäten, Probleme mit Strukturen, Leistungsprobleme, Schulwechsel und Schulsuspendierung männliche Patienten mehr Auffälligkeiten aufwiesen als weibliche (siehe Tab. 3).

Tab. 4 zeigt die prozentuelle Verteilung der einzelnen schulischen Auffälligkeiten innerhalb der verschiedenen Störungskategorien. Tab. 5 vergleicht jene Kategorien und zeigt, dass PatientInnen mit externalisierenden Störungen mehr Verhaltensprobleme in der Schule aufwiesen, als PatientInnen ohne einer Störung. PatientInnen mit sowohl internalisierenden als auch externalisierenden Störungen zeigten mehr Verhaltensprobleme, Verwicklung in einen Raufhandel und Probleme mit Strukturen im Vergleich zu

\begin{tabular}{|c|c|c|c|}
\hline $\begin{array}{l}\text { Gesamtstichprobe } \\
n / N\end{array}$ & $\begin{array}{l}\text { Jungen } \\
n / N\end{array}$ & $\begin{array}{l}\text { Mädchen } \\
n / N\end{array}$ & $p$ \\
\hline $66 / 128(51,6 \%)$ & $38 / 55(69,1 \%)$ & $28 / 73(38,4 \%)$ & $0,001^{* *}$ \\
\hline $34 / 127(26,8 \%)$ & $25 / 54(46,3 \%)$ & $9 / 73(12,3 \%)$ & $0,000^{* *}$ \\
\hline 49/127 (38,6\%) & $33 / 54(61,1 \%)$ & $16 / 73(21,9 \%)$ & $0,000^{* *}$ \\
\hline $72 / 127(56,7 \%)$ & $29 / 54(53,7 \%)$ & $43 / 73(58,9 \%)$ & 0,559 \\
\hline $53 / 127(47,1 \%)$ & $34 / 54(63,0 \%)$ & $19 / 73(26,0 \%)$ & $0,000^{* *}$ \\
\hline $43 / 127(33,9 \%)$ & $20 / 54(37,0 \%)$ & $23 / 73(31,5 \%)$ & 0,515 \\
\hline $82 / 127(64,6 \%)$ & $41 / 54(75,9 \%)$ & $41 / 73(56,2 \%)$ & $0,021^{*}$ \\
\hline $46 / 127(36,2 \%)$ & $25 / 54(46,3 \%)$ & $21 / 73(28,8 \%)$ & $0,042^{*}$ \\
\hline $22 / 127(17,3 \%)$ & $18 / 54(33,3 \%)$ & $4 / 73(5,5 \%)$ & $0,000^{* *}$ \\
\hline $61 / 126(48,4 \%)$ & $26 / 53(49,1 \%)$ & $35 / 73(47,9 \%)$ & 0,902 \\
\hline
\end{tabular}

PatientInnen ohne einer Störung. PatientInnen mit ausschließlich externalisierenden Störungen, sowie externalisierenden und internalisierenden Störungen, zeigten öfter Verhaltensprobleme, Autoritätsprobleme, Probleme mit Strukturen, Leistungsprobleme, gerieten öfter in einen Raufhandel, wurden öfter von der Schule suspendiert und hatten öfter Kontakt mit SchulpsychologInnen als PatientInnen mit ausschließlich internalisierenden Störungen.

Die Analyse der intra- und extrafamiliären Belastungen ergab, dass Jungen häufiger Opfer von extrafamiliärem körperlichem Missbrauch waren $(24,5 \%$ vs. $9,7 \%), \chi 2(1)=4,979 ; p=0,026$. Im Gegensatz dazu zeigte sich, dass Mädchen öfter von sexuellem Missbrauch betroffen sind (3,7\% vs. $20 \%), \chi 2(1)=7,204 ; p=0,007$. Alle anderen intra- und extrafamiliären Variablen erwiesen sich als nicht signifikant unterschiedlich hinsichtlich Geschlecht, internalisierender, externalisierender oder beider Formen der psychischen Störungen. 
Tab. 4 Anzahl schulischer Auffälligkeiten innerhalb der verschiedenen Störungskategorien

\begin{tabular}{|c|c|c|c|c|c|}
\hline Schulische Auffälligkeiten & $\begin{array}{l}\text { Gesamtstichprobe } \\
n / N\end{array}$ & $\begin{array}{l}\text { Keine Störung } \\
n / N\end{array}$ & $\begin{array}{l}\text { Internalisierende } \\
\text { Störungen } \\
n / N\end{array}$ & $\begin{array}{l}\text { Externalisierende Störungen } \\
n / N\end{array}$ & $\begin{array}{l}\text { Ext. und int Störungen } \\
n / N\end{array}$ \\
\hline Verhaltensprobleme & $62 / 124(50 \%)$ & $3 / 13(23,1 \%)$ & $10 / 46(21,7 \%)$ & $12 / 18(66,7 \%)$ & $37 / 47(78,7 \%)$ \\
\hline Raufhandel & $30 / 123(24,4 \%)$ & $1 / 12(8,3 \%)$ & $3 / 46(6,5 \%)$ & $6 / 18(33,3 \%)$ & $20 / 47(42,6 \%)$ \\
\hline Autoritätsprobleme & $45 / 123(36,6 \%)$ & $3 / 12(25 \%)$ & $7 / 46(15,2 \%)$ & $10 / 18(55,6 \%)$ & $25 / 47(53,2 \%)$ \\
\hline Probleme mit KollegInnen & $67 / 123(54,5 \%)$ & $4 / 12(33,3 \%)$ & $26 / 46(56,5 \%)$ & $11 / 18(61,1 \%)$ & $26 / 47(55,3 \%)$ \\
\hline Probleme mit Strukturen & $51 / 123(41,1 \%)$ & $3 / 12(25 \%)$ & $7 / 46(15,2 \%)$ & $11 / 18(61,1 \%)$ & $30 / 47(63,8 \%)$ \\
\hline Probleme mit Fehlzeiten & $42 / 123(34,1 \%)$ & $6 / 12(50 \%)$ & $12 / 46(26,1 \%)$ & $6 / 18(33,3 \%)$ & $18 / 47(38,3 \%)$ \\
\hline Leistungsprobleme & $79 / 123(64,2 \%)$ & $8 / 12(66,7 \%)$ & $21 / 46(45,7 \%)$ & $14 / 18(77,8 \%)$ & $36 / 47(76,6 \%)$ \\
\hline Schulwechsel & $43 / 123(35 \%)$ & $3 / 12(25 \%)$ & $12 / 46(26,1 \%)$ & $8 / 18(44,4 \%)$ & $20 / 47(42,6 \%)$ \\
\hline Schulsuspendierung & $20 / 123(16,3 \%)$ & $1 / 12(8,3 \%)$ & $0 / 46(0 \%)$ & $4 / 18(22,2 \%)$ & $15 / 47(31,9 \%)$ \\
\hline Kontakt mit SchulpsychologInnen & $60 / 122(49,2 \%)$ & $5 / 12(41,7 \%)$ & $17 / 46(37 \%)$ & $11 / 18(61,6 \%)$ & $27 / 47(58,7 \%)$ \\
\hline
\end{tabular}

Tab. 5 Vergleich schulischer Auffälligkeiten zwischen den verschiedenen Störungskategorien

\begin{tabular}{|c|c|c|c|c|c|c|}
\hline Schulische Auffälligkeiten & $\begin{array}{l}\text { Keine Störung } \\
\text { vs. } \\
\text { Internalisierende } \\
\text { Störungen } \\
\mathrm{Chi}^{2}(p)\end{array}$ & $\begin{array}{l}\text { Keine Störung } \\
\text { vs. } \\
\text { Externalisierende } \\
\text { Störungen } \\
\text { Chi }^{2}(p)\end{array}$ & $\begin{array}{l}\text { Keine Störung } \\
\text { vs. } \\
\text { Ext. \& Int. Störun- } \\
\text { gen } \\
\text { Chiं }^{2}(p)\end{array}$ & $\begin{array}{l}\text { Internalisierende } \\
\text { vs. } \\
\text { Externalisierende } \\
\text { Störungen } \\
\mathrm{Chi}^{2}(p)\end{array}$ & $\begin{array}{l}\text { Internalisierende } \\
\text { vs. } \\
\text { Ext. \& Int. Störun- } \\
\text { gen } \\
\text { Chi }^{2}(p)\end{array}$ & $\begin{array}{l}\text { Externalisierende } \\
\text { vs. } \\
\text { Ext. \& Int. Störungen } \\
\text { Chi }^{2}(p)\end{array}$ \\
\hline Verhaltensprobleme & $0,11(1)^{\mathrm{a}}$ & $5,743(0,017)^{*}$ & $14,190(0,000)^{a, * *}$ & $11,576(0,001)^{\star \star}$ & $30,199(0,000)^{\star *}$ & $1,02(0,346)^{\mathrm{a}}$ \\
\hline Raufhandel & $0,049(1)^{\mathrm{a}}$ & $2,516(0,193)^{a}$ & $4,883(0,041)^{a, *}$ & $7,696(0,012)^{a, *}$ & $16,213(0,000)^{\star \star}$ & $0,461(0,597)$ \\
\hline Autoritätsprobleme & $0,638(0,417)^{\mathrm{a}}$ & $2,738(0,098)$ & $3,047(0,081)$ & $20,792(0,003)^{a, \star *}$ & $14,854(0,000)^{\star \star}$ & $0,029(0,864)$ \\
\hline Probleme mit KollegInnen & $2,049(0,152)$ & $2,222(0,136)$ & $1,849(0,174)$ & $0,112(0,738)$ & $0,014(0,907)$ & $0,178(0,673)$ \\
\hline Probleme mit Strukturen & $0,638(0,417)^{\mathrm{a}}$ & $3,772(0,052)$ & $5,848(0,016)^{\star}$ & $13,48(0,000)^{\star \star}$ & $22,932(0,000)^{\star \star}$ & $0,041(0,839)$ \\
\hline Probleme mit Fehlzeiten & $2,543(0,161)^{\mathrm{a}}$ & $0,833(0,458)^{\mathrm{a}}$ & $0,542(0,521)^{\mathrm{a}}$ & $0,336(0,562)$ & $1,586(0,208)$ & $0,183(0,711)$ \\
\hline Leistungsprobleme & $1,681(0,195)$ & $0,455(0,678)^{\mathrm{a}}$ & $0,497(0,479)^{\mathrm{a}}$ & $5,338(0,02)^{*}$ & $9,382(0,002)^{\star \star}$ & $0,01(1)^{\mathrm{a}}$ \\
\hline Schulwechsel & $0,006(1)^{\mathrm{a}}$ & $1,172(0,442)^{a}$ & $1,238(0,334)^{\mathrm{a}}$ & $2,029(0,154)$ & $2,793(0,095)$ & $0,019(0,089)$ \\
\hline Schulsuspendierung & $3,901(0,207)^{\mathrm{a}}$ & $1(0,622)^{\mathrm{a}}$ & $2,69(0,151)^{\mathrm{a}}$ & $10,904(0,005)^{a, * *}$ & $17,504(0,000)^{a, \star \star *}$ & $0,591(0,442)$ \\
\hline $\begin{array}{l}\text { Kontakt mit Schulpsycho- } \\
\text { logInnen }\end{array}$ & $0,09(0,752)^{\mathrm{a}}$ & $1,094(0,296)$ & $1,116(0,291)$ & $3,067(0,08)^{\star}$ & $4,356(0,037)^{*}$ & $0,031(0,86)$ \\
\hline \multicolumn{7}{|c|}{$\begin{array}{l}{ }^{*} \mathrm{Ch}^{2} \text {-Test ist signifikant } p<0,05 \\
{ }^{*} \mathrm{Ch}^{2} \text {-Test ist signifikant } p<0,01 \\
{ }^{a} \text { Exakter Test nach Fisher bei erwartetem } n<5\end{array}$} \\
\hline
\end{tabular}

In Bezug auf psychische Vorbehandlungen und selbstgefährdendes Verhalten befanden sich 92 $(72,4 \%)$ PatientInnen der Gesamtstichprobe vor Erstvorstellung an der Ambulanz in psychischer Behandlung.

Mädchen wiesen in ihrer Vergangenheit deutlich häufiger Selbstmordversuche $(20,8 \%$ vs. $3,7 \%$; $\chi 2(1)=7,757 ; p=0,005)$ und selbstverletzendes Verhalten $(62,5 \%$ vs. $31,5 \% ; \chi 2(1)=11,879 ; p=0,001)$ auf als Jungen. PatientInnen mit internalisierenden Störungen neigten eher zu selbstverletzendem Verhalten als diejenigen mit externalisierenden Störungen (58,7\% vs. $27,8 \%) ; \chi 2(1)=4,947 ; p=0,026$. Im Weiteren hatten PatientInnen mit sowohl internalisierenden als auch externalisierenden Störungen beinahe doppelt so häufig ein psychisch erkranktes Familienmitglied als jene mit lediglich externalisierenden Erkrankungen $(81 \%$ vs. $44,7 \%) ; \chi 2(1)=7,997 ; p=0,005$.

In Bezug auf die Freizeitgestaltung ergab die Analyse, dass Jungen $(n=30 ; 55,6 \%)$ deutlich häufiger ihre Freizeit mit der Nutzung eines Computers verbringen als Mädchen $(n=18 ; 25,4 \%) ; \chi 2(1)=11,829 ; p=0,001$.
Die häufigste positive Drogenanamnese fand sich in der Gruppe jener PatientInnen, welche sowohl an internalisierenden als auch externalisierenden Störungen litt $(n=11 ; 24,4 \%)$. Am seltentsten konsumierten PatientInnen mit ausschließlich internalisierenden Störungen illegale Substanzen ( $n=2 ; 4,3 \%)$.

\section{Diskussion}

Ähnlich der MHAT-Study [1], welche in der klinischen Stichprobe eine Punkträvalenz jeglicher Erkrankungen von $88,06 \%$ fand, waren in unserer ambulanten Kohorte 86,9\% aller Kinder und Jugendlichen psychisch erkrankt.

In unserer Kohorte wurden Mädchen und Jungen gleich häufig erstvorstellig, auch in Bezug auf das Alter bei Erstvorstellung fand sich kein signifikanter Unterschied. In der Gruppe der PatientInnen ab 14 Jahren fand sich, ähnlich zu vergangenen Studien, ein vermehrtes Auftreten von Suizidalität, Depression und/ oder Dysthymie. 
Im Einklang mit der MHAT-Study zeigte sich, dass Jungen häufiger an Verhaltensstörungen und Aufmerksamkeits- und/oder Hyperaktivitätsstörungen leiden.

Die Prävalenz der einzelnen Störungen im Vergleich mit der klinischen Stichprobe der MHAT-Study zeigte, dass in unserer Kohorte die Depression und Dysthymie $(31,9 \%$ vs. $49,25 \%)$ sowie die Essstörungen (8\% vs. 34,33\%) seltener auftraten. Angst und Panikstörungen sowie Suizidalität zeigten sich in etwa gleich häufig $(31,2 \%$ vs. $31,34 \%$ und $26,8 \%$ vs. $21,51 \%)$. Es fand sich eine erhöhte Prävalenz in der vorliegenden Studie im Vergleich mit der MHAT-Study bei den Verhaltensstörungen $(27,5 \%$ vs. $6,58 \%)$ und den trauma-assoziierten Störungen (25,4\% vs. $13,38 \%$; [1]). Der gravierende Unterschied im Auftreten der Verhaltensstörungen ergibt sich vermutlich daraus, dass diese Störungen häufiger ambulant und seltener stationär behandelt werden. Im Weiteren sind PatientInnen mit Verhaltensstörungen jünger als jene mit anderen Erkrankungen. Daher ist zu erwarten, dass stationäre Aufenthalte für jene PatientInnen eher vermieden werden. Da in unserer Stichprobe ausschließlich ambulante Kontakte untersucht wurden, liegt die Vermutung nahe, dass dadurch mehr PatientInnen mit Verhaltensstörungen eingeschlossen wurden.

Mädchen litten in unserer Kohorte im Vergleich zu Jungen öfter an einer trauma-assoziierten Störung, Angst- und/oder Panikstörung, Essstörungen, Suizidalität, selbstverletzendem Verhalten oder einer depressiven Störung. In Bezug auf trauma-assozierte Störungen zeigen auch frühere Studien eine häufigere Entwicklung von PTBS-Symptomen bei Mädchen, vor allem nach gewaltsamen Erlebnissen [20].

In Übereinstimmung mit der Literatur litten Jungen in unserer Studie signifikant öfter unter externalisierenden Erkrankungen [21]. In Bezug auf schulische Auffälligkeiten zeigte sich, dass Jungen in sieben von zehn Kategorien mehr Probleme aufwiesen als Mädchen. PatientInnen mit externalisierenden Störungen hatten im Vergleich zu PatientInnen mit keiner oder lediglich einer internalisierenden Störungen ebenfalls öfters mit schulischen Problemen zu kämpfen. Dieser Unterschied spiegelt die Schwierigkeit wider, externalisierende Störungen und das Regelschulsystem miteinander zu vereinbaren. In Summe besteht ein beunruhigender Zusammenhang zwischen Psychopathologie und Schulproblemen, der insbesondere männliche Kinder und Jugendliche betrifft. Dieses Ergebnis führt zu der Frage, ob eventuelle Entwicklungsschädigungen durch schulisches Versagen unvermittelt in Kauf genommen werden, ohne Rücksicht auf die eigentlichen Bedürfnisse einzelner Schülergruppen.

Mehr als die Hälfte der PatientInnen dieser Studie gaben an, den Verlust eines Familienmitglieds erlebt zu haben. Die Trennung von oder der Verlust eines Elternteils führt zu erhöhten Raten an Depression, Angst und Substanzmissbrauch und stellt als solches einen Ansatz für Präventionsmaßnahmen zur Verhinderung psychischer Erkrankungen nach einem solchen Verlust dar [22]. Sexueller Missbrauch betraf mit $20 \%$ deutlich häufiger Mädchen als Jungen mit 3,7\%. Es muss an dieser Stelle erwähnt werden, dass die vermutete Dunkelziffer zu sexuellen Übergriffen groß ist. Die UNICEF schätzt, dass sieben von zehn weltweiten Missbrauchsopfern nie Hilfe aufsuchen [23]. Männliche Kinder und Jugendliche, andererseits, wurden häufiger Opfer extrafamiliären Schlagens.

Die psychiatrische Eigenanamnese der Gesamtstichprobe zeigte, dass $72,4 \%$ aller PatientInnen bereits in psychischer Behandlung waren. Diese Zahlen sind aus medizinökonomischer Sicht erfreulich, da die Ambulanz der kinder- und jugendpsychiatrischen Universitätsklinik somit für einen Großteil der PatientInnen nicht die ersten Anlaufstelle darstellte.

Mindestens ein psychisch erkranktes Familienmitglied fand sich bei $68,1 \%$ der PatientInnen. Eine Erkrankung eines oder beider Elternteile ist sowohl auf Grund der genetischen Belastungen als auch möglicher erzieherischer Dysfunktionen hochrelevant [24]. Es erscheint daher unerlässlich, durch familiäre psychische Erkrankung vorbelastete Kinder vor weiteren Stressoren zu schützen. Ein Ansatz dafür ist die Kooperation mit der Erwachsenenpsychiatrie und Miteinbeziehung der Erwachsenen in die Behandlung der Kinder.

Die Freizeitgestaltung unserer Kohorte zeigte, dass $55,6 \%$ der Jungen häufige Freizeitgestaltung unter Nutzung eines Computers angaben, verglichen mit 25,4\% der Mädchen. Problematisches Online Gaming fand sich in vergangenen Studien häufiger bei jungen Männern, allerdings zeigten Mädchen in neueren Studien oftmals erhöhten Social-Media Gebrauch [25-27]. Die Folgen dieses Verhaltens beinhalten beispielsweise den erhöhten Konsum von zuckerhaltigen Getränken, geringere physische Aktivität und Schlafsstörungen, welche wiederum Auslöser für die Entwicklung weiterer psychischer Symptome sein können [28, 29].

Wie bereits bei den schulischen Auffälligkeiten waren auch bei Drogenkonsum jene PatientInnen mit einer Kombination aus internalisierenden und externalisierenden Störungen stärker gefährdet als jene mit lediglich internalisierenden Störungen. Internalisierende Störungen zeigten sich bereits in Studien mit delinquenten PatientInnen als ungünstige Variante in Bezug auf Verhaltensproblematik und bieten somit Grundlage für weitere Studien [30].

Innerhalb des Studienzeitraums dieser Studie wurden alle ambulant erstvorstelligen PatientInnen untersucht, welche die Einschlusskriterien erfüllten. Somit bot sich eine gute Möglichkeit, Prävalenzraten von Psychopathologien im ambulanten Behandlungssetting der Universitätsklinik Salzburg zu untersuchen. Die PatientInnen wurden mithilfe wissenschaftlich erprobter Testverfahren untersucht. Auf Grund der Besonderheiten der Stichprobe psychisch erkrankter Kinder- und Jugendlicher konnten dennoch nicht al- 
le Variablen gleichermaßen erhoben werden. Daher ergaben sich für die einzelnen Analysen verschiedene Anzahlen an ProbandInnen. Insgesamt wurden bei $13,1 \%$ der vorstelligen PatientInnen keine psychischen Störungen duch das MINI-KID festgestellt. Diese PatientInnen haben bei der systematischen Erörterung psychopathologischer Auffälligkeiten, trotz Inanspruchnahme der Terminambulanz, den durch den Test vorgegebenen Grenzwert für keine der erwähnten Erkrankung überschritten und konnten somit keiner Kategorie zugeordnet werden.

Das MINI-KID ist ein umfangreiches Untersuchungstool, welches einige Krankheiten erfasst, die auf Grund der niedrigen Prävalenzzahlen nicht in die vorliegende Studie einflossen (Bipolare Störungen, Tourette Syndrom, tiefgreifende Entwicklungsstörungen).

Weitere Limitationen der vorliegenden Studie betreffen die Informationsgewinnung, welche ausschließlich durch Befragung der PatientInnen stattfand. Überdies handelt es sich um eine rein retrospektive Studie ohne Verlaufskontrollen, deren Datenerhebung im Rahmen von Routineuntersuchungen ohne Zweitbegutachtung stattfand. Obwohl alle erstmalig vorstelligen PatientInnen untersucht wurden, ergab sich eine relativ geringe Anzahl an ProbandInnen. Eine Attritionsanalyse wurde auf Grund fehlender Informationen nicht durchgeführt.

Bereits publizierte Daten werden durch unsere Studie ergänzt, indem sie die Ergebnisse zur Prävalenz psychopathologischer Erkrankungen bei Kindern und Jugendlichen um Daten zu ambulant geplant vorstelligen PatientInnen in Österreich ergänzt [31]. Vor allem Jungen zeigen sich in Bezug auf schulische Auffälligkeiten als besonders vulnerabel. Darüber hinaus zeigen die vorliegenden Daten, dass der Großteil der PatientInnen vor Erstvorstellung an der Ambulanz bereits psychologische, psychotherapeutische oder psychiatrische Hilfe in Anspruch genommen hat. Dies sind erfreuliche Zahlen, welche auf ein niedrigschwelliges System in der Begutachtung psychopathologischer Erstmanifestationen hindeuten. Erhöhte Wachsamkeit jenen gegenüber, die von psychosozialen Belastungen betroffen sind, könnte dazu beitragen, frühzeitig und präventiv die Entwicklung von psychischen Symptomen zu identifizieren und vor dem Ausbruch einer psychiatrischen Erkrankung zu behandeln.

Funding Open access funding provided by Paracelsus Medical University.

\section{Einhaltung ethischer Richtlinien}

Interessenkonflikt C. Skrivanek, E. Marte, K. Winds, L. ThunHohenstein und B. Plattner geben an, dass kein Interessenkonflikt besteht.

Ethische Standards Einverständniserklärungen der Studienteilnehmer waren durch die retrospektive Natur der Untersuchung nicht erforderlich.
Open Access Dieser Artikel wird unter der Creative Commons Namensnennung 4.0 International Lizenz (http:// creativecommons.org/licenses/by/4.0/deed.de) veröffentlicht, welche die Nutzung, Vervielfältigung, Bearbeitung, Verbreitung und Wiedergabe in jeglichem Medium und Format erlaubt, sofern Sie den/die ursprünglichen Autor(en) und die Quelle ordnungsgemäß nennen, einen Link zur Creative Commons Lizenz beifügen und angeben, ob Änderungen vorgenommen wurden.

\section{Literatur}

1. Wagner G, Zeiler M, Waldherr K, Philipp J, Truttmann S, DürW, Treasure JL, Karwautz AFK. Mental health problems in Austrian adolescents: a nationwide, two-stage epidemiological study applying DSM-5 criteria. Eur Child Adolesc Psychiatry. 2017;26:1483-99.

2. Meltzer H, Gatward R, Goodman R, Ford T. The mental health of children and adolescents in. Great Britain: London: The stationary Office; 1999.

3. Anda RF, Felitti VJ, Bremner JD, Walker JD, Whitfield C, Perry BD, Dube SR, Giles WH. The enduring effects of abuse and related adverse experiences in childhood. Eur Arch Psychiatry Clin Neurosci. 2006;256:174-86.

4. Finkelhor D, ShattuckA, Turner HA, Hamby SL. The lifetime prevalence of child sexual abuse and sexual assault assessed inlate adolescence. JAdolesc Heal. 2014;55:329-33.

5. Hardt J, Sidor A, Nickel R, Kappis B, Petrak P, Egle UT. Childhood adversities and suicideattempts:Aretrospective study. J Fam Violence. 2008;23:713-8.

6. BundesministeriumfürGesundheitundFrauen. Suizidund Suizidprävention in Österreich. 2017. https:/ / www.google.

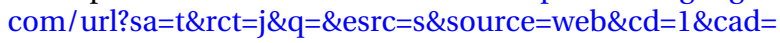
rja\&uact=8\&ved=2ahUKEwj7_tvH14HiAhXj14sKHWoGAYg QFjAAegQIABAC\&url=https\%3A\%2F\%2Fbroschueren service.sozialministerium.at\%2FHome\%2FDownload\%3F publicationId\%3D660\&usg=AOvVaw290QwdVHk_ZW9 smTVASTKp.Zugegriffen 13 Dez2018.

7. Wichstrøm L. Predictors of adolescent suicide attempts: A nationally representative longitudinal study of Norwegian adolescents. J Am Acad Child Adolesc Psychiatry. 2000;39:603-10.

8. Bielas H, Barra S, Skrivanek C, Aebi M, Steinhausen HC, Bessler C, Plattner B. The associations of cumulative adverse childhood experiences and irritability with mental disorders in detained male adolescent offenders. Child Adolesc Psychiatry Ment Health. 2016;10:1-10.

9. Finlay-Jones R, Brown GW. Types of stressful life event and the onset of anxiety and depressive disorders. Psychol Med. 1981;11:803-15.

10. Hovens JGFM, Wiersma JE, Giltay EJ, Van Oppen P, Spinhoven P, Penninx BWJH, Zitman FG. Childhood life events and childhood trauma in adult patients with depressive, anxiety and comorbid disorders vs. controls. Acta Psychiatr Scand. 2010;122:66-74.

11. Andersen SL, Teicher MH. Stress, sensitive periods and maturational events in adolescent depression. Trends Neurosci. 2008;31:183-91.

12. Derks EM, Hudziak JJ, Boomsma DI. Why more boys than girls with ADHD receive treatment: A study of Dutch twins. Twin Res Hum Genet. 2007;10:765-70.

13. BergerE,AichhornW, MaxF, Fiala PreinsbergerS, Leixnering W, Mangold B, Spiel G, Tatzer G, Thun-Hohenstein L. Kinder- und Jugendpsychiatrische Versorgung Österreich Neuropsychiatr. 2006;20:86-90.

14. Thun-Hohenstein L. Die Versorgungssituation psychisch auffälliger und kranker Kinder und Jugendlicher in Öster- 
reich.In: Kerbl R, Thun-Hohenstein L, VavrikK, Waldhauser F, Herausgeber. Kindermedizin - Werte versus Ökonomie. Wien: Springer; 2008. S. 163-173.

15. American Psychiatric Association American Psychiatric Association. Diagnostic and statistical manual of mental disorders, fourth edition, text revision. Am Psychiatr Assoc. 2000.https://doi.org/10.1176/appi.books.9780890423349.

16. Sheehan DV, Lecubier Y, Sheehan KH, Amorim P, Janavs J, Weiller E, Hergueta T, Baker R, Dunbar GC. The Mini-International Neuropsychiatric Interview (M.I.N.I): The development and validation of a structured diagnostic psychiatric interview for DSM-IV and ICD-10. J Clin Psychiatry. 1998;59:22-33.

17. Bauer SM, Steiner H, Feucht M, Stompe T, Karnik N, Kasper S, Plattner B. Psychosocial background in incarcerated adolescents from Austria, Turkey and former Yugoslavia. Psychiatry Res. 2011;185:193-9.

18. Fisher RA. On the interpretation of $\chi 2$ from contingency tables, and the calculation of P. J Royal Stat Soc. 1922;85:87-94.

19. Field A. Discovering statistics using SPSS. London: SAGE; 2009. https://doi.org/10.1234/12345678.

20. Seedat S, van Nood E, Vythilingum B, Stein DJ, Kamlner D. School survey of exposure to violence and posttraumatic stress symptoms in adolescents. South African J Child Adolesc Ment Heal. 2000;12:38-44.

21. Ihle W, Esser G, Schmidt MH, Blanz B. Die Bedeutung von Risikofaktoren des Kindes- und Jugendalters für psychsiche Störungen von der Kindheit bisins früheErwachsenenalter. Kindh Entwickl.2002;11,pp:201-11.

22. Otowa T, York TP, Gardner CO, Kendler KS, Hettema JM. The impact of childhood parental loss on risk for mood, anxiety and substance use disorders in a population-based sample of male twins. Psychiatry Res. 2014;220:404-9.

23. UNICEF. Hidden In Plain Sight. A statistical analysis of violence against children. UNICEF 2014. https://www. unicef.org/publications/index_74865.html.Zugegriffen20 Dez2018.

24. Cadoret RJ, Cain CA, Crowe RR. Evidence for gene-environment interaction in the development of adolescent antisocial behavior. Behav Genet. 1983;13:301-10.

25. Reiner I, Tibubos AN, Hardt J, Müller K, Wölfling K, Beutel ME. Peer attachment, specific patterns of internet use and problematic internet use in male and female adolescents. Eur Child Adolesc Psychiatry. 2017;26:1257-68.

26. RumpfH, MeyerC, KreuzerA, John U.PrävalenzderInternetabhängigkeit ( PINTA) Bericht an das Bundesministerium für Gesundheit. 2011. https://www.google.com/url?sa=t\& $r c t=j \& q=\& e s r c=s \&$ source $=$ web $\& c d=1 \&$ ved $=2$ ahUKEwj0ro7 mlIHiAhXtAhAIHa6rCZYQFjAAegQIARAC\&url=http\%3A\% 2F\%2Fwww.fachportalsucht-nrw.de\%2Ftl_files\%2Fimages \%2Fpages\%2FPDFs\%2FPINTA-Bericht-Endfassung 280611.pdf\&usg=AOvVaw1BrwkVxfh8pIq2Q2RK6Tnv.Zugegriffen 4 Jan 2018.

27. Müller KW, Wölfling K, Beutel ME, Stark B, Quiring O, Aufenanger S, Schemer C, Weber M, Reinecke L. Insights into aspects behind internet-related disorders in adolescents: The interplay of personality and symptoms of adjustment disorders. J Adolesc Heal. 2018;62:234-40.

28. Kenney EL, Gortmaker SL. United States adolescents' television, computer, videogame, smartphone, and tablet use: associations with sugary drinks, sleep, physical activity, and obesity. J Pediatr. 2017;182:144-9.

29. Marino C, Vieno A, Lenzi M, Borraccino A, Lazzeri G. Computer use, sleep difficulties, and psychological symptoms among school-aged children: The mediating role of sleep difficulties. Int J School Health 2017;4(1):e32921. https:// doi.org/10.17795/intjsh-32921

30. Plattner B, Aebi M, Steinhausen HC, Bessler C. Psychopathologischeund komorbideStörungen inhaftierterJugendlicher in Österreich Implikationen für einen jugendpsychiatrischen Versorgungsauftrag im Strafvollzug. Z Kinder Jugendpsychiatr Psychother. 2011;39, pp:231-42.

31. Fuchs M, Karwautz A. Epidemiologie psychischer Störungen bei Kindern und Jugendlichen: Eine narrative Übersichtsarbeit unter Berücksichtigung österreichischer Daten. Neuropsychiatrie. 2017;31:96-102.

32. World Health Organisation. ICD-10 Version:2010. In: World Heal. Organ 2010. https://icd.who.int/browse10/2010/en. Zugegriffen 4 Nov 2018.

Hinweis des Verlags Der Verlag bleibt in Hinblick auf geografische Zuordnungen und Gebietsbezeichnungen in veröffentlichten Karten und Institutsadressen neutral. 